Instituto Internacional de Investigación y Desarrollo Tecnológico Educativo INDTEC, C.A.

DOI: https://doi.org/10.29394/scientific.issn.2542-2987.2017.2.3.6.118-137

OAI-PMH: http://www.indteca.com/ojs/index.php/Revista Scientific/oai

\title{
Competencias Gerenciales en el Logro de la Calidad Educativa en las Instituciones del Nivel Primario
}

Autora: Yelitza del Carmen Morillo Terán Universidad Nacional Experimental Rafael María Baralt, UNERMB yecarmar 72@hotmail.com Zulia, Venezuela

\section{Resumen}

La presente investigación tiene como propósito analizar la importancia de las competencias gerenciales en el logro de la calidad educativa en las instituciones del nivel primario ubicadas en la parroquia Marcelino Briceño del municipio Baralt. Para ello se sustenta teóricamente en aportes de autores como Programa AME (2012), Robbins y De Cenzo (2008), entre otros. Metodológicamente será de tipo analítico, con diseño de campo, no experimental, transeccional. La población está conformada por 74 individuos, discriminados de la siguiente manera: 12 directivos, 62 docentes pertenecientes a las instituciones seleccionadas. Se utilizará la encuesta como técnica de recolección de datos mediante un cuestionario con escala tipo Likert, estructurado por cinco alternativas. La validez se obtendrá a través del juicio de cinco expertos, la confiabilidad se calculará mediante el coeficiente Alfa de Cronbach. Los resultados obtenidos permitirán dar respuesta a los objetivos de la investigación por medio de la elaboración de conclusiones concretas, así como recomendaciones dirigidas a aportar alternativas de solución a la problemática planteada.

Palabras clave: competencias gerenciales; calidad educativa; educación primaria.

Fecha de Recepción: 20-08-2016

Fecha de Aceptación: 18-09-2016 


\title{
Management Skills in Achieving Educational Quality in Primary Institutions
}

\begin{abstract}
This research aims to analyze the importance of managerial skills in achieving quality education in primary level institutions located in the parish Marcelino Briceño Baralt municipality. For it is theoretically based on contributions from authors such as Programa AME (2012), Robbins and De Cenzo (2008), among others. Methodologically will be analytic, with field design, not experimental, transeccional. The population is made up of 74 individuals, discriminated as follows: 12 directors, 62 teachers from the selected institutions. The survey and data collection technique will be used through a questionnaire with Likert scale, structured by five alternatives. The validity will be obtained through the trial of five experts, the reliability is calculated by Cronbach Alfa. The results allow to respond to the research objectives through the development of specific conclusions and recommendations to provide alternative solutions to the issues raised.
\end{abstract}

Keywords: managerial skills; quality of education; primary education.

Date Received: 20-08-2016

Date Acceptance: 18-09-2016 


\section{Introducción}

La educación es un elemento fundamental para el desarrollo de la sociedad en todos sus niveles: económico, político, cultural, social, pues es la encargada de formar a los ciudadanos y ciudadanas para asumir los retos futuros en la búsqueda de una mejor calidad de vida. Desde esta perspectiva, es indispensable que quienes estén a cargo de tal misión sean personas capacitadas académicamente para propiciar la construcción de los conocimientos de éstos.

Desde esa perspectiva, se considera que la buena marcha de las instituciones educativas depende en gran parte de la gestión directiva desarrollada por los gerentes, quienes poseen competencias conducentes al éxito de la organización, siendo necesario para ello, promoverla, así como definir una línea gerencial en la aplicación de las funciones administrativas las cuales permitan optimizar el desempeño laboral de todos sus miembros, en consecuencia, alcanzar los objetivos propuestos.

Ahora bien, en el ámbito mundial, la educación es considerada como elemento fundamental para el desarrollo social, económico, político, cultural, forma los recursos humanos indispensables para participar, responsable y activamente, en los cambios, transformaciones que demandan las sociedades. El logro de tal cometido requiere disponer de instrumentos idóneos los cuales orienten, contribuyan a alcanzar los objetivos de progreso de los sistemas educativos, tal como los roles del gerente educativo.

Asimismo, a nivel de Latinoamérica, se han venido suscitando profundas transformaciones para los diferentes ámbitos del quehacer social, siendo la educación la más influenciada por ellas, razón por la cual es fundamental la constante actualización de quienes participan: directivos, docentes. En este sentido, corresponde al gerente escolar velar porque el personal docente desarrolle su función educadora de una manera óptima, para alcanzar las metas propuestas y con ello la calidad educativa. 
Por ello, la Organización de las Naciones Unidas para la Educación, la Ciencia y la Cultura (UNESCO, 2007: 41), enuncia que las naciones de América Latina trabajan con el propósito construir y consolidar su desarrollo económico, fortalecer sus estructuras basándose en la educación como fundamento para el futuro", razón por la cual esta ha experimentado múltiples transformaciones en virtud de ser el ente que tiene a su cargo la formación de individuos íntegros, adaptados a los cambios para el desarrollo de países como México, Argentina, Chile, entre otros.

Visto de esta forma, cabe señalar que el director a través del ejercicio de sus competencias gerenciales es el responsable de la calidad de la educación impartida desde sus organizaciones escolares a través del manejo de sus procesos como: planificación, organización, dirección, control, los cuales son decisivos para el desarrollo de las actividades académicas, pues esto permite evidenciar el esfuerzo y la actuación del docente, traduciéndose éste en un mejoramiento de su desempeño pedagógico, en consecuencia una mayor calidad educativa.

Cabe señalar que en los últimos años, Venezuela ha experimentado numerosos cambios en las diferentes áreas del quehacer nacional e internacional, siendo los más evidentes los acaecidos en materia educativa, entre ellos: mayor inclusión social de personas, así como sectores sociales desfavorecidos, incremento del número de servicios o instituciones educativas para todos los niveles y modalidades, búsqueda de calidad/pertinencia social de los procesos educativos bajo la concepción humanista de una educación cuyo centro de interés es el desarrollo humano, entre otros.

\subsection{Formulación del Problema}

¿Qué importancia tienen de las competencias gerenciales en el logro de la calidad educativa en las instituciones del nivel primario ubicadas en la parroquia Marcelino Briceño del municipio Baralt? 


\section{Teoría y Conceptos}

\subsection{Objetivos de la Investigación}

\subsubsection{Objetivo General}

Analizar la importancia de las competencias gerenciales en el logro de la calidad educativa en las instituciones del nivel primario ubicadas en la parroquia Marcelino Briceño del municipio Baralt.

Se hace necesario hacer mención, sustentar este artículo se hizo necesario realizar una revisión bibliográfica y documental, de la cual surgió un conjunto de estudios referidos realizados para los cambios propuestos, que persiguen alcanzar la calidad educativa, la acción directiva en los centros educativos en el país, responde fundamentalmente a un modelo burocrático, gerencialista, que, en opinión de Silva (2010: 12), concibe a las escuelas como estructuras formales articuladas en torno a relaciones explícitas de autoridad, subordinación y como instituciones con procedimientos uniformes con énfasis en los subprocesos administrativos de planificación, organización, dirección, control.

Este cambio en la gerencia permite colocar la institución educativa en condiciones de incorporarse al proceso de reformas planteadas por el Estado, ajustándola a las metas institucionales, en fin, dar otra organización a la escuela, basada en la idea de una gestión directiva con la cual se dé respuesta a las múltiples funciones del plantel, así como al tipo de docentes requeridos, y en consecuencia, a la clase de colegio esperado.

Dentro de ese contexto, el Programa de Actualización del Maestro de Escuela (AME, 2012) plantea que la calidad de un centro educativo requiere de los directivos: una concepción estratégica, integral, global de la función de la organización; el establecimiento de metas asociadas al desempeño institucional esperado, a partir del diagnóstico objetivo de las necesidades de sus estudiantes, los recursos disponibles, así como los necesarios, el entorno inmediato; la evaluación permanente de la gestión, tanto directiva como 
docente involucrando a los diferentes niveles en el logro de resultados parciales y totales.

Sin embargo, señala Medina (2009), se observa la inexistencia de una formación gerencial adecuada con la aplicación de una planificación normativa, caracterizada por la rigidez, centralización de la organización, la ausencia de delegación de autoridad, así como carencia de liderazgo. Asimismo, Castillo (2008) señala que en los países latinoamericanos y, concretamente, en Venezuela existen debilidades relacionadas con la gestión directiva de las instituciones escolares relacionadas con el cumplimiento de sus funciones, por cuanto los gerentes no poseen las competencias necesarias para llevar a cabo un proceso estratégico, el cual permita generar cambios en sus escuelas a partir de una adecuada dirección.

Esta realidad se observa en las instituciones del nivel primario ubicadas en la parroquia Marcelino Briceño del municipio Baralt, en donde, según información emanada de la Coordinación Escolar de esta jurisdicción (2013) el director se concreta en cumplir con las actividades rutinarias, planificaciones repetitivas año tras año, cumplimiento de horarios, tramitación de documentos, pero sin ejercer una verdadera gerencia desde su cargo jerárquico.

Lo anteriormente planteado podría ocasionar un descenso de la calidad educativa de los egresados de los planteles, pues sin una buena gerencia toda organización escolar disminuye su nivel de efectividad. Por lo anteriormente expuesto, en esta investigación se analiza la importancia de las competencias gerenciales en el logro de la calidad educativa en las instituciones del nivel primario ubicadas en la parroquia Marcelino Briceño del municipio Baralt.

La revisión bibliográfica realizada por la investigadora arrojó una serie de estudios relacionados con el trabajo de investigación, los cuales aportan evidencias que, a juicio de la investigadora, son pertinentes con las variables propuestas, sirviendo de soporte al mismo. Entre ellos las investigaciones Briñez (2011), Camacho (2011), quienes destacan la similitud de las 
problemáticas en las instituciones educativas relacionadas por el dominio de las competencias gerenciales por parte de los directivos escolares; así como las de Moscote (2012), De Yúnez (2009) donde se destaca la relevancia de fortalecer la calidad educativa para el progreso educativo y la mejora de la calidad de vida de los ciudadanos.

Por tanto, las competencias gerenciales pueden definirse como la aptitud de un gerente para desempeñar sus funciones con base en los requerimientos de calidad específicos de su campo laboral. Esta aptitud se logra con la adquisición y desarrollo de conocimientos, habilidades, capacidades expresadas en el saber, hacer, saber hacer, es decir, la capacidad real para lograr un objetivo o un resultado en un contexto dado.

Al respecto, Mitrani y otros (2009: 24) afirman que competencia "es una característica subyacente en una persona que está causalmente relacionada con una actuación exitosa en un puesto de trabajo". En ese sentido, se consideran capacidades complejas que poseen distintos grados de integración y se manifiestan en una gran variedad de situaciones en los distintos ámbitos de la vida humana, personal, social.

Visto de esa forma, es pertinente entender que las competencias gerenciales son comportamientos observables, pero también subjetivos en cuanto que la percepción de los mismos depende del observador, por tal razón, cuando se evalúan las competencias se requiere un procedimiento más cuidadoso que el de los objetivos. Éstas pueden ser: genéricas, laborales, profesionales.

Las competencias genéricas, de acuerdo con Tobón (2006) las competencias genéricas se caracterizan por: (a) aumentar las posibilidades de empleabilidad, al permitirle a las personas cambiar fácilmente de un trabajo a otro; (b) favorecer la gestión, consecución y conservación del empleo; (c) permitir la adaptación a diferentes entornos laborales; (d) no estar ligadas a una ocupación en particular; (e) adquirirse mediante procesos sistemáticos de 
enseñanza y aprendizaje; (f) su adquisición y desempeño pueden evaluarse de manera rigurosa.

En cuanto a las competencias laborales son una manera de describir: (a) lo que una persona es capaz de hacer; (b) la persona en la cual puede juzgarse si el trabajo está bien hecho; (c) las condiciones en las cuales la persona debe demostrar su aptitud; (e) los tipos de evidencia necesarios para tener la seguridad de haber realizado el trabajo de manera consistente, con base en un conocimiento efectivo y no como producto de la casualidad.

Finalmente, las competencias profesionales, son definidas por Leboyer (2005) como una cualidad humana que se configura como síntesis dialéctica en la integración funcional del saber (conocimientos diversos), saber hacer (habilidades, hábitos, destrezas y capacidades), saber ser (valores, actitudes) los cuales son movilizados en un desempeño idóneo a partir de los recursos personológicos del sujeto que le permiten saber estar en un ambiente socioprofesional y humano acorde con las características, exigencias de las situaciones que enfrenta relativas a la profesión. Una manera de demostrar el dominio de competencias gerenciales por el directivo es el ejercicio adecuado de sus funciones básicas: planificación, dirección, organización, control.

Para Robbins y De Cenzo (2008: 78) la planificación es la función gerencial que "define objetivos y decide, tanto los recursos como las tareas necesarias para alcanzarlos adecuadamente". Esta función, en las instituciones educativas, significa mirar hacia adelante, ver el futuro para decidir las acciones a realizar, elaborar nuevos planes, así como ayudar a los docentes a realizar las actividades necesarias para enfrentar los retos permanentes de la educación.

En relación a la organización, Melinkoff (2006: 10) expresa "la organización es una función proyectiva de la empresa que se complementa con la dirección, pues solamente a través de ello es factible lograr el objetivo que por sí sola no alcanzaría". De acuerdo con lo anteriormente expuesto, la 
organización es una función gerencial realizada por el directivo escolar mediante la cual este asigna a los componentes de su personal: docentes, personal administrativo, obrero, determinadas responsabilidades específicas según su relación jerárquica.

Asimismo, Soto (2009: 38) plantea "la acción de la dirección es un proceso llevado a cabo para imprimir orden, hacer crecer la institución como sistema, asociándose con la planificación, la organización”, concibiéndola como la función encargada de instrumentar el plan, usar los recursos organizados en operaciones, tanto reales como efectivas, para lograr los objetivos establecidos.

La cuarta función el control, en opinión de Rincón y Núñez (2008), es una función gerencial mediante la cual el producto de una actividad se compara con los estándares establecidos. Como proceso le permite al directivo escolar evaluar y corregir el desempeño de las acciones del personal para asegurarse que los planes de la institución se estén llevando en la forma prevista. En las instituciones educativas, señalan las autoras mencionadas, es imprescindible controlar las siguientes áreas: propósito, estructura, relaciones, recompensa-castigo.

Ahora bien, partiendo del consenso de que la educación es un derecho humano fundamental, así como un bien público irrenunciable, la Oficina Regional de Educación para América Latina y el Caribe dependiente de la OREALC/UNESCO (Organización de las Naciones Unidas para la Educación, 2007) concibe la calidad educativa como un medio para que el ser humano se desarrolle plenamente como tal, por cuanto gracias a ella crece, se fortalece como persona, como especie la cual contribuye al desarrollo de la sociedad, transmitiendo, compartiendo tanto sus valores como su cultura, basada en cinco dimensiones esenciales: equidad, relevancia, pertinencia, eficacia, eficiencia. 
La equidad, según García (2009), debe basarse en una postura igualitaria, pues debe implantar la regulación centralizada del sistema para otorgar recursos compensatorios de la situación original de cada institución, sin aceptar la idea de que cada uno tenga proyectos propios, los cuales faciliten el alcance de estos recursos de forma autónoma. Se interpreta entonces que la orientación igualitaria es la manejada desde el Sistema Educativo Venezolano, con el propósito de terminar con las diferencias encontradas en las diferentes regiones del país.

La relevancia es un componente esencial de los sistemas educativos que pretenden la calidad, refiriéndose al qué y para qué de la educación. Desde la primera, implica lograr el pleno desarrollo del ser humano, lo cual significa fortalecer el desarrollo de sus capacidades. Por ello, para considerar relevante lo que se enseña, debe estar orientado al cumplimiento de las finalidades de la educación en un contexto espacio-temporal determinado.

La pertinencia demanda la inclusión del otro como legítimo e igual, respetando, valorando los universos simbólicos, en consecuencia, los paradigmas que los sostienen. No asumir diferencias culturales en las poblaciones atendidas transforma estas diferencias culturales iniciales en desigualdades de acceso al conocimiento, de aprendizajes, éxito escolar, oportunidades sociales, por tanto, construye y legitima la exclusión social.

En cuanto a la eficacia, Schmelkes (2007), la define como la capacidad del sistema de cumplir con los objetivos que le han sido asignados, incluye los aspectos de cobertura, permanencia, promoción, aprendizaje real. Desde esa perspectiva, el componente de la eficacia corresponde de manera significativa con la orientación administrativa de la calidad, que juega de igual forma un papel importante junto con el resto de los elementos del concepto.

Asimismo, define la eficiencia como el óptimo empleo de los recursos para mejores resultados", es decir, cuáles son los costos de lograr los objetivos planteados. No debe ser vista como un elemento economicista, sino como una 
condición que respete los derechos de todos los ciudadanos a recibir educación y aproveche los recursos con los cuales se cuenta. Dentro de los factores que influyen en el logro de la calidad educativa existen tres factores primordiales que contemplan todos los componentes antes mencionados: gestión directiva, competencias docentes, desempeño académico de los estudiantes.

Según Chiavenato (2008: 262) la gestión directiva se define como "las actividades que efectúan las organizaciones al planificar, organizar, dirigir, controlar a objeto de utilizar los recursos humanos, físicos y financieros, para alcanzar objetivos comúnmente relacionados con beneficios económicos, materiales, de servicio, entre otros".

De acuerdo con lo anterior, la gestión directiva requiere de un gerente efectivo, es decir, un directivo con capacidad para actuar eficiente y eficazmente, lo cual se logra con base en suficientes competencias, habilidades para dirigir con responsabilidad, establecer nuevas relaciones interpersonales apoyándose tanto en la autoridad como en el compromiso en las instituciones educativas.

La competencia docente corresponde a la parte normativa, funcional del trabajo académico que le permite desempeñarse adecuadamente en el contexto de las prácticas educativas concretas de este campo profesional. Estas se expresan en desempeños genéricos, los cuales corresponden a las características de las funciones y acciones propias de prácticas docentes específicas del quehacer cotidiano del docente dependiendo del nivel donde éste se ubique.

Finalmente, el desempeño académico de los estudiantes es considerado por Palacios y Andrade (2007) está asociada al acopio sistemático de datos cuantitativos y cualitativos que permite formular juicios de valor acerca de las características o variables medidas, para así determinar si los cambios propuestos en los objetivos de aprendizaje se están realizando en 
los educandos, para poder expresarlo mediante la calificación asignada por el profesor o el promedio obtenido por ellos.

\section{Metodología}

\subsection{Enfoque epistemológico}

Esta investigación se ubica en el enfoque cuantitativo positivista el cual estará dado por el diagnóstico de la situación real para analizar la importancia de las competencias gerenciales en el logro de la calidad educativa en las instituciones del nivel primario ubicadas en la parroquia Marcelino Briceño del municipio Baralt., del cual se obtendrá una medida aritmética de las variables con sus dimensiones, indicadores e ítems. Estos resultados conjuntamente con los enfoques teóricos presentados, permitirán el análisis crítico, interpretativo de los datos, así como la redacción de conclusiones y recomendaciones pertinentes.

\subsection{Tipo de investigación}

La presente investigación será analítica sustentándose en lo expuesto por Finol y Camacho (2006:56) quienes plantean que este tipo de estudios reinterpreta los datos en función de los criterios teóricos presentados en el marco referencial dependiendo de los objetivos de análisis.

\subsection{Diseño de la investigación}

La presente investigación, se categoriza como un estudio de campo, no experimental, transeccional, pues se refiere al método que se emplea cuando los datos de interés se recogen en forma directa de la realidad donde se desarrolla la problemática, en un solo momento y sin intervenir en el comportamiento de las variables. 


\subsection{Población y muestra}

\subsubsection{Población}

Camacho (2011:158) define la población como "el conjunto de todos los elementos a los cuales se refiere la investigación". En este orden de ideas, la unidad de análisis de la presente investigación estará conformada por 12 directivos y 62 docentes de las escuelas del nivel primario ubicadas en la parroquia Marcelino Briceño del municipio Baralt, considerándose por tanto trabajar con el censo poblacional.

\subsubsection{Muestra}

Según Chávez (2007: 164) la muestra es "una porción representativa de la población, que permite generalizar sobre esta, los resultados de una investigación". De igual forma, Finol y Camacho (2006: 62) la definen como "un grupo pequeño de la población (sujeto, documentos, objetos), por lo tanto, tiene sus mismas características"

Para los efectos de esta investigación se tomará la población total, por ser esta muy reducida y estadísticamente manejable. Esta decisión se sustenta en lo planteado por Bernal (2009: 168) quien expresa que "las poblaciones pequeñas deben tomarse en su totalidad como una forma de garantizar la consecución de datos confiables para el estudio".

\subsection{Técnica e instrumento de recolección de datos}

En la recolección de los datos necesarios para la descripción de la variable se seleccionará la encuesta. Asimismo, como instrumento de recolección de datos se utilizará un cuestionario estructurado, según una escala tipo Likert modificada la cual establece proposiciones con selección múltiple, según lo señala Sánchez (2007), con cinco (05) alternativas de respuestas: siempre (S), casi siempre (CS), algunas veces, (AV), casi nunca 
$(\mathrm{CN})$, nunca $(\mathrm{N})$, que servirán para obtener las respuestas emitidas por la población participante a las afirmaciones propuestas en las mismas.

\subsection{Validez y confiabilidad del instrumento}

\subsubsection{Validez}

La validez para Finol y Camacho (2006: 86), "constituye el proceso mediante el cual se estima: a) la medida en que las conclusiones representan la realidad empírica; b) si los constructos elaborados por los investigadores representan las categorías de la experiencia humana"

Para tal fin, el instrumento diseñado será sometido a un proceso de validación a través del juicio de cinco expertos, quienes lo revisarán en cuanto a forma y contenido, para considerar la pertinencia del mismo con los objetivos del estudio. Por tanto, se elaborará una guía de validación de instrumentos, la cual se entregará a los especialistas antes señalados, quienes realizarán la lectura/corrección del cuestionario a aplicar, a los efectos de cumplir con la validación, así como arrojar las observaciones pertinentes con el objeto de efectuar las correcciones necesarias.

\subsubsection{Confiabilidad}

En cuanto a la confiabilidad del instrumento de la presente investigación, esta se obtendrá con la aplicación del Coeficiente de Cronbach mediante el paquete estadístico SPSS 17.0, el cual se utiliza cuando el instrumento posee varias alternativas de respuestas, y se representa mediante la siguiente fórmula:

$$
\mathrm{Rtt}=\mathrm{k} / \mathrm{k}-1\left[1-\sum \mathrm{Si}^{2} / \mathrm{St}^{2}\right]
$$

Donde:

$\mathrm{K}=$ número de ítems

1 = es una constante

Si2 = Varianza de los puntajes de cada ítem 
St2 = Varianza de los puntajes totales

Al operacionalizar en la fórmula anterior, los resultados obtenidos de una prueba piloto aplicada a una población con características similares a las del estudio, se obtendrá un cociente el cual deberá ubicarse en el intervalo comprendido entre 0,8 y 1 , ambos inclusive para poder ser catalogado como altamente confiable.

\subsection{Técnica de análisis de los datos}

Para tabular los datos se aplicarán tablas de doble entrada donde se ubicaron los datos de la variable con sus respectivos indicadores e ítems, colocando para ello en el margen izquierdo el número de integrantes de la población censal conformada por 12 directivos y 62 docentes de las escuelas del nivel primario ubicadas en la parroquia Marcelino Briceño del municipio Baralt. Mientras, en el margen superior se colocaron los ítems correspondientes a cada instrumento.

La medición de la variable se realizará mediante los cálculos de estadística descriptiva frecuencias, para obtener las frecuencias absolutas y relativas de los datos aportados por la muestra como una forma de evidenciar la manifestación de los indicadores en el comportamiento de la variable en el trabajo, las cuales se mostrarán en cuadros.

\subsection{Procedimiento de la investigación}

Luego de realizar la identificación y descripción del objeto de estudio seleccionado para desarrollar la presente investigación, se ubicaron, seleccionaron, las fuentes de información necesarias, tanto documentales como bibliográficas, las cuales fueron consultadas, analizadas, para obtener un marco teórico que fundamentara el estudio, así como obtener los indicadores de las variables de estudio en atención a sus dimensiones. 
Posteriormente, se continuó con el proceso investigativo para determinar los aspectos relacionados con los métodos, diseño y técnica de recolección de datos, las cuales permitirán obtener bases sólidas para elaborar el cuestionario, de acuerdo con los objetivos formulados, el cual se someterá al juicio de expertos, así como a una prueba piloto para conocer su coeficiente de confiabilidad. Los resultados obtenidos permitirán la formulación de conclusiones concretas, así como recomendaciones pertinentes dirigidas a ofrecer alternativas de solución a la problemática detectada.

\section{Conclusiones}

Sin lugar a dudas, como investigadora verso las siguientes conclusiones:

De acuerdo con tal postura, la relación sujeto-objeto en el proceso de producción del conocimiento es relevante señalar que la investigación se hizo dinámica, por cuanto la variable no se establece de una vez sino a través de sucesivas aproximaciones, resultando diferente de acuerdo a la actitud del sujeto investigación frente al objeto estudiado.

De esa manera, en correspondencia con los objetivos propuestos, tipifica como analítica, de campo, en razón del análisis que se realizará a la importancia de las competencias gerenciales en el logro de la calidad educativa en las instituciones del nivel primario ubicadas en la parroquia Marcelino Briceño del municipio Baralt, sustentándose en un diseño no experimental, transeccional de campo, el cual se aplicará a una población constituida por 12 directivos y 62 docentes pertenecientes a los centros educativos antes mencionados

Para la recolección de datos se utilizará como técnica la encuesta mediante un instrumento tipo escala multi-opcional de respuestas estructurado con cinco alternativas de respuestas y 45 ítems, las cuales permitirán obtener las respuestas emitidas por la población participante a las afirmaciones propuestas en las mismas, el cual será validado por el juicio de 7 expertos, 
mientras la confiabilidad se obtendrá por la aplicación del coeficiente Alfa de Cronbach a los resultados de una prueba piloto suministrada a una población con características similares a las del estudio.

Los datos obtenidos con la aplicación del instrumento a las unidades de información serán analizados, estadística y valorativamente, lo cual permitirá obtener resultados fiables, que posibilitarán la redacción de conclusiones y recomendaciones para atender la problemática detectada.

\section{Referencias}

Bernal, C. (2009). Metodología de la Investigación. Prentice Hall. Bogotá. Briñez, R. (2011). "Competencias gerenciales del director para la administración del talento humano". Trabajo de grado. Maestría Gerencia Educativa. Universidad Rafael Belloso Chacín. Maracaibo.

Camacho, Y. (2011). "Competencias gerenciales del personal directivo y el desempeño laboral de los docentes en escuelas básicas". Trabajo de grado. Maestría Gerencia Educativa. Universidad Rafael Urdaneta. Maracaibo.

Castillo, B. (2008). "Estilo gerencial de los directores en el comportamiento organizacional en las unidades educativas de Educación Media Diversificada". Tesis Doctoral. Doctorado en Ciencias de la Educación. Universidad Dr. Rafael Belloso Chacín. Maracaibo.

Chávez, N. (2007). Introducción a la Investigación Educativa. Gráfica González. Maracaibo.

Chiavenato, I. (2008). "Administración en los nuevos tiempos". Editorial McGraw-Hill. México.

Coordinación Escolar del municipio Baralt (2013). "Informe de gestión". Mene Grande. Estado Zulia. 
De Yúñez, F. (2009). "El desempeño docente desde una perspectiva humanística hacia la optimización de la calidad educativa para el siglo XXI en los institutos universitarios de tecnología". Tesis Doctoral. Universidad de Carabobo. Valencia.

García, V. (2009). "Calidad de la educación, trabajo y libertad”. Ediciones Dossat S.A. Madrid.

Finol, M. y Camacho, H. (2006). "El proceso de investigación científica". Fondo Editorial Universidad del Zulia. Maracaibo.

Leboyer, L. (2005) “Administración de recursos humanos”. Editorial Prentice Hall. México

Medina, O. (2009). "Gestión directiva y trabajo en equipo en el subsistema Educación Básica, Media General”. Trabajo de Grado. Maestría en Gerencia Educativa. Universidad Rafael Urdaneta. Maracaibo.

Melinkoff, A. (2006). "Gerencia”. Editorial Paidós. Buenos Aires.

Mitrani, A. y otros (2009). "Las competencias". Ediciones Deusto. Bilba.

Moscote, F. (2012). "Indicadores de calidad educativa en instituciones de educación básica". Trabajo de grado. Maestría Gerencia Educativa. Universidad Rafael Urdaneta. Maracaibo.

OREALC/UNESCO (2007). "Educación de calidad para todos: un asunto de derechos humanos". Ediciones UNESCO. Santiago de Chile.

Palacios, A. y Andrade, F. (2007). "Psicología evolutiva”. Editorial Morata. Madrid.

Programa AME (2012). "Formación de competencias básicas directivas para la gestión de centros educativos". Fundación Cisneros. Caracas.

Rincón, J. y Núñez, M. (2008). "Supervisión y Gerencia”. Universidad Nacional Abierta. Caracas.

Robbins, S. y De Cenzo, F. (2008). "Fundamentos de administración". Editorial Prentice Hall. México. 
Schmelkes, S. (2007). "Educación para la vida". Editorial Aique. Buenos Aires.

Sánchez, H. (2007). La solución de problemas como un campo de concurrencia de distintas teorías en Psicología. Revista colombiana de psicología, (16), 147-162, e-ISSN: 2344-8644. Recuperado de: http://www.revistas.unal.edu.co/index.php/psicologia/article/view/1012

Silva, F (2010). "Desempeño gerencial y gestión directiva en educación intercultural de educación básica primaria". Trabajo de Grado. Maestría en Gerencia Educativa. Universidad Rafael Urdaneta. Maracaibo.

Soto, P. (2009). "Gerencia en las organizaciones escolares". Editorial Trillas. México.

Tobón, S. (2006). "Formación basada en competencias". Editorial Ecoe. Bogotá.

UNESCO (2007). "Eficacia escolar y factores asociados en América Latina y el Caribe". Santiago de Chile. 


\section{Yelitza del Carmen Morillo Terán \\ e-mail: yecarmar 72@hotmail.com}

Nació en San Rafael de El Tigre, Municipio Baralt,

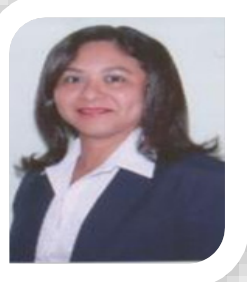
Estado Zulia, el 17 de Septiembre de 1972. Estudiante de la Universidad Nacional Experimental "RAFAEL MARÍA BARALT" en el DOCTORADO EN EDUCACIÓN. Estudios de postgrado: Universidad "Valle de Momboy". Título obtenido: ESPECIALISTA EN EVALUACIÓN EDUCACIONAL. Estudio de Pregrado: U.N.E.R.M.B. Titulo Obtenido: LICENCIADA EN EDUCACIÓN, ÁREAS: Lenguaje y Ciencias Naturales. Ha participado en cursos, talleres y simposios sobre Análisis Institucional UNERMB. 1994. Implantación del Programa de Estudio de la Educación Religiosa Escolar I y II Etapa de la Educación Básica. Ministerio de Educación 1996. Fortaleciendo la Familia Diócesis de Cabimas. Sec. De Pastoral Social y Ministerio de la Familia. Duración 28 horas. Julio 1999. Capacitación y Actualización sobre el Nuevo Diseño Curricular, dirigido a los Docentes en servicio de la I Etapa de Educación Básica. Ministerio de Educación, Cultura y Deporte. Julio 2000. Operador en Computadora Registrado en el ministerio de Educación. Duración 48 horas. 2001 Importancia y efecto de la Droga en los niños, niñas y adolescentes. Ministerio de Educación, Cultura y Deporte. Enero 2003. Experiencia laboral: Escuela "Hilarión Alberto Viloria". Docente de Aula. Desde: 02-09-1995. Hasta 2013. Facilitadora de la Misión Sucre, Desde: 04-09- 2006 Hasta 07-2013. Actualmente Docente con Función Supervisora desde el 03/06/2013 hasta la presente fecha.

El contenido de este manuscrito se difunde bajo una Licencia de Creative Commons ReconocimientoNoComercial-Compartirlgual 4.0 Internacional 